\title{
Involvement of spindles in memory consolidation is slow wave sleep-specific
}

\author{
Roy Cox, ${ }^{1}$ Winni F. Hofman, and Lucia M. Talamini \\ Department of Psychology, University of Amsterdam, Weesperplein 4, 1018 XA Amsterdam, The Netherlands
}

\begin{abstract}
Both sleep spindles and slow oscillations have been implicated in sleep-dependent memory consolidation. Whereas spindles occur during both light and deep sleep, slow oscillations are restricted to deep sleep, raising the possibility of greater consolidation-related spindle involvement during deep sleep. We assessed declarative memory retention over an interval containing a nap and determined spindle density for light and deep sleep separately. In deep sleep, spindle density was considerably higher and showed a strong and robust positive correlation with retention. This relation was absent for light sleep, suggesting that the potentiating effects of spindles are tied to their co-occurrence with slow oscillations.
\end{abstract}

The importance of sleep for declarative memory consolidation has gained increasing support over the years. Non-rapid eye movement (NREM) sleep and its signature oscillatory events, sleep spindles and slow oscillations, have been shown to be of particular relevance in this respect (Born et al. 2006). Sleep spindles are transient, waxing and waning, rhythmic brain signals with a center frequency between 11 and $16 \mathrm{~Hz}$. They are generated by the interplay of cells of the thalamic reticular nucleus and thalamocortical relay neurons (Steriade 2006), although their duration is also affected by neocortical feedback (Bonjean et al. 2011).

The role of spindles in learning-related processes is supported by their plasticity-inducing properties. In particular, spiking patterns underlying spindles are capable of potentiating cortical synapses (Rosanova and Ulrich 2005). Furthermore, tasks requiring learning (compared with no or less-intense encoding) lead to increases in various spindle measures (Gais et al. 2002; Schmidt et al. 2006). In addition, spindle activity correlates with memory retention over sleep (Clemens et al. 2005, 2006; Van der Helm et al. 2011) and predicts performance in a directed-forgetting paradigm (Saletin et al. 2011), suggesting spindle involvement in memory consolidation processes.

While spindles occur throughout NREM sleep, spindles during slow-wave sleep (SWS) might be of special relevance for memory consolidation. Indeed, a few reports suggest that the benefits of sleep for declarative memory performance are specifically related to slow $(<1.5 \mathrm{~Hz})$ oscillations, the hallmark oscillatory events during SWS (Marshall et al. 2006, 2011). In these studies, slow oscillation activity during sleep was either boosted or suppressed with transcranial electrical stimulation, enhancing or worsening subsequent memory performance, respectively.

Importantly, slow oscillations have a naturally occurring grouping influence on spindles (Mölle et al. 2002; Clemens et al. 2007), and such focusing of spindle rhythms into the active slow oscillation up-state increases after a learning episode (Mölle et al. 2009). This phase locking is combined with an even more finegrained nesting of hippocampal ripples inside single troughs of sleep spindles (Clemens et al. 2011). It is during these ripples that the classic "replay" of waking neural patterns mostly occurs, both in hippocampal and neocortical regions (O'Neill et al. 2010). As such, the temporal coupling of hippocampal ripples, sleep spindles, and slow oscillations might reflect a hippocampo-cortical di-

\footnotetext{
${ }^{1}$ Corresponding author.

E-mail r.cox@uva.nl.

Article is online at http://www.learnmem.org/cgi/doi/10.1101//m.026252.112.
}

alogue underlying system-level memory consolidation during sleep (Buzsáki 1996; Born et al. 2006).

Considering the purported role of slow oscillations in memory consolidation, it is noteworthy that previous reports linking spindle measures to memory retention do not distinguish between sleep stages with and without these slow waves. Furthermore, some of these studies (Clemens et al. 2005, 2006) are unable to firmly distinguish between the role of spindles per se and sleep-duration related effects. Therefore, the current study set out to investigate whether the involvement of sleep spindles in memory retention is SWS-specific and whether such involvement is unrelated to a more general influence of sleep.

For this purpose participants were exposed to a memory task with a retention interval containing a nap. Spindles were detected separately in NREM sleep epochs marked by sleep spindles and a relative absence of slow oscillations (light or stage 2 sleep) and NREM episodes involving both types of oscillation (deep sleep or SWS). We focused on spindle density (number of spindles per minute), to avoid confounds introduced by individual differences in total sleep duration and time spent in particular sleep stages. Spindle density, as well as sleep architectural parameters and power in different frequency bands were correlated with memory retention over sleep. Since it is largely unknown whether spindle characteristics differ between light sleep and SWS, we also compared spindle density and several other spindle parameters between these sleep stages.

Nineteen subjects participated in the experiment (mean age \pm SD: $21.3 \pm 5.2$, four male). They were self-reported good sleepers, who habitually slept at least $7 \mathrm{~h}$ per night between 11 PM and 9 AM. They had no prior history of neurological, psychiatric, or sleep disorders and had not previously seen the movie from which the stimulus was drawn (see below). All had normal or corrected-to-normal vision. Participants were asked to refrain from the use of alcohol or other drugs from $2 \mathrm{~d}$ before the experiment until the experimental sessions ended. All subjects provided written informed consent, and the local ethics committee approved the experiment.

On the night prior to the experiment, subjects were deprived of the last third of their normal sleep period to enhance the likelihood of a full-cycle nap containing sufficient amounts of light sleep and deep sleep on the next (experimental) day. Compliance with this protocol was assessed via actimetry. The next morning, sleepiness was assessed using the Dutch version of the Stanford Sleepiness Scale (SSS). Then, at 9.30 AM, subjects were instructed to attentively watch a film fragment (6-min extract from "March 
of the Penguins," Luc Jacquet). Subjects were informed that they would have to answer questions about the film. Memory for film content was tested with 11 multiple-choice questions (three alternative answers and "I don't know") both 5 min after the film and $11 \mathrm{~h}$ later (8.30 PM). Subjects were explicitly requested not to guess. A different questionnaire was used for each retrieval session and the order of the questionnaires was counterbalanced over subjects. Questions pertained to both perceptual ("What color eyes did the penguin chicks have?") and conceptual aspects of the film ("In what month did the ice begin to melt?"). Importantly, questions were constructed such that correct answers based on semantic memory were highly unlikely. Retention of film content was expressed as (100\% $\times$ delayed recall/immediate recall).

During the retention interval all subjects took a nap (onset 12 AM) under polysomnographic registration (EEG, EOG, and EMG sampled at $200 \mathrm{~Hz}$ with a 16-channel Monet system [Medcare]). Electrode impedance was kept below $5 \mathrm{k} \Omega$. EEG was recorded from F3, F4, C3, C4, O1 (10/20 system), referenced to linked mastoids, band-pass filtered between 0.03 and $70 \mathrm{~Hz}$, and notch filtered at $50 \mathrm{~Hz}$. After entering stage 2 sleep, participants were allowed to sleep for a maximum of $2 \mathrm{~h}$. If they failed to reach stage 2 sleep within an hour, or if they failed to sleep for at least $1 \mathrm{~h}$, they were excluded from the experiment.

Sleep stages were visually scored according to standard criteria (Rechtschaffen and Kales 1968) using REMbrandt software (Medcare) and an epoch size of $30 \mathrm{sec}$. In addition, the frequency content of the EEG between 0.5 and $50 \mathrm{~Hz}$ was analyzed using a fast Fourier transform-based spectral analysis (4-sec Hamming window, $50 \%$ overlap, $0.25 \mathrm{~Hz}$ bin size). For each bin, the average power per 30-sec epoch was computed. Next, epochs containing stage 2 sleep and SWS were averaged separately. Finally, bins were merged and power in the slow oscillation $(0.5-1.5 \mathrm{~Hz}), \delta$ $(1.5-4 \mathrm{~Hz}), \theta(4-8 \mathrm{~Hz}), \alpha(8-12 \mathrm{~Hz}), \sigma(11-16 \mathrm{~Hz}), \beta(15-25 \mathrm{~Hz})$, and $\gamma(25-50 \mathrm{~Hz})$ frequency bands was determined.

Sleep spindles were identified by a dynamic detection algorithm implemented in MATLAB (2007b, the Mathworks, Natick), closely resembling one described earlier (Ferrarelli et al. 2007). Briefly, for each subject and for channels C3 and C4 separately, the EEG signal was zero-phase band-pass filtered between 11 and $16 \mathrm{~Hz}$ with a 4 th order IIR filter, rectified, and its envelope was computed. Next, the average envelope amplitude during spindle-containing sleep stages $(2,3$, and 4$)$ was determined. Whenever the envelope crossed an upper threshold of 3.5 times the average envelope, a potential spindle was identified. The beginning and end of a potential spindle were marked as the time

Table 1. Sleep architecture parameters $(n=16)$

\begin{tabular}{lcr}
\hline Sleep stage & Time (minutes) & \multicolumn{1}{c}{ SD } \\
\hline 1 & 16.9 & 20.2 \\
2 & 42.6 & 19.3 \\
3 & 15.9 & 8.2 \\
4 & 28.2 & 22.5 \\
REM & 19.4 & 10.5 \\
SWS $(3+4)$ & 44.1 & 24.1 \\
NREM $(2+3+4)$ & 86.6 & 21.9 \\
Total sleep & 122.9 & 8.1 \\
\hline
\end{tabular}

points where the envelope fell below one-third of the upper threshold. A sleep spindle was required to have a minimum duration of 500 msec. For light and deep sleep separately, spindle density (spindle count per minute), average spindle amplitude, and average spindle duration were determined. Finally, spindle measures from C3 and C4 were averaged. Our threshold settings were chosen to achieve optimal agreement between spindle events detected by the algorithm and spindles visually identified by an experienced sleep scorer (WFH).

Two subjects were outliers with respect to memory retention $(Z>3.29)$ and were excluded from further analyses. One additional subject spent $>80 \%$ of sleep time in stage 1 sleep, and was removed as well. Therefore, our total number of included cases is 16. Table 1 displays general sleep architectural parameters across all included subjects. All participants spent time in SWS and all reached REM sleep. Average retention score was $94 \pm$ $23 \%$ (range: $43 \%-125 \%$ ).

Interestingly, we detected a significantly higher spindle density during deep as opposed to light sleep (light sleep: $6.6 \pm 1.8$; deep sleep: $10.6 \pm 1.9 ; t_{(15)}=-5.1, P<0.001$ ) (Fig. 1). Average spindle amplitudes and durations, however, did not differ from light to deep sleep (spindle amplitude: $5.7 \pm 2.5 \mu \mathrm{V}$ in light sleep vs. $5.6 \pm 2.6 \mu \mathrm{V}$ in deep sleep, $P>0.25$; spindle duration: $1135 \pm$ 136 msec in light sleep vs. $1102 \pm 91$ msec in deep sleep, $P>0.25$ ).

With regard to spindle density and memory retention, no significant relation was detected for stage 2 sleep $(r=-0.25$, $P=0.35$, Pearson correlation). In contrast, we found a strong and highly significant positive correlation between spindle density during SWS and memory retention $(r=0.68, P=0.004)$ (Fig. 2). To test whether this association was more pronounced during deep sleep than during light sleep, the pertaining correlation coefficients were contrasted directly (Meng et al. 1992), revealing a significantly stronger link between memory retention and spindle density during SWS than during stage 2 sleep $(Z=2.3, P=0.011$, one-sided).

Given the subjects' partly sleep-deprived state, it is possible that the aforementioned correlations were influenced by pre-nap sleepiness. Therefore, partial correlations between spindle density and memory retention were calculated, controlling for sleepiness as indexed by the SSS. Again, no correlation was found between spindle density during stage 2 sleep and memory retention $(r=-0.31, P=0.27)$, while for SWS this relation remained strikingly significant $(r=0.67, P=0.007)$. Also, the correlation coefficient was significantly higher for deep sleep than for light sleep $(Z=2.2, P=0.013$, one-sided).

Next, we recalculated correlations while controlling for initial (pre-sleep) memory performance to rule out possible 
A

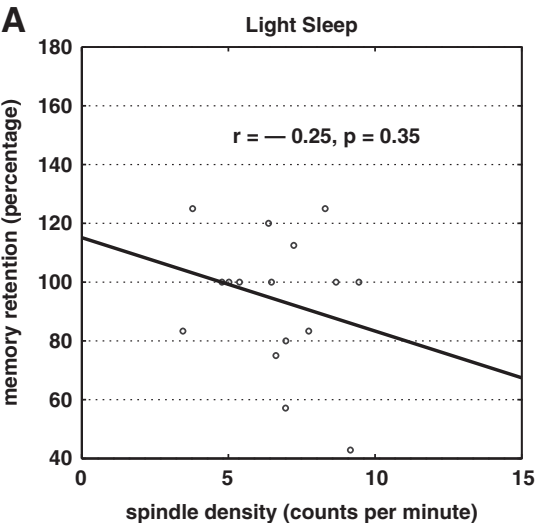

B

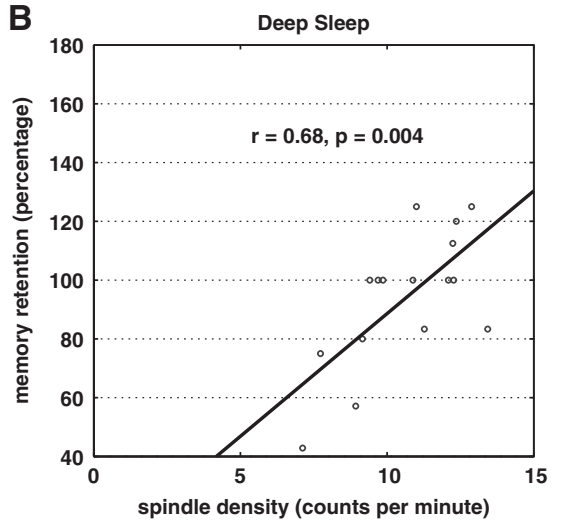

Figure 2. Correlation between memory retention and spindle density for light sleep $(A)$ and for deep sleep $(B)(n=16)$.

influences of encoding success on subsequent spindle density. Once again, we found no significant correlation during stage 2 $(r=-0.18, P=0.51)$, while a reliable link during SWS was maintained $(r=0.58, P=0.025)$. And again, the correlation was stronger for deep sleep $(Z=1.7, P=0.046$, one-sided).

Memory retention did not robustly correlate with power in any frequency band. We observed a correlation between memory retention and power in the slow oscillation band during light sleep ( $r=0.53, P=0.04)$ only when controlling for pre-nap sleepiness. However, this association did not survive the Bonferroni-adjusted $\alpha$ level correcting for multiple frequency bands $(0.05 / 7=0.007)$. No other frequency band, including $\sigma$, showed a significant correlation between power and memory retention, in either light or deep sleep (all $P s>0.08$ ).

Finally, none of the sleep architectural parameters correlated significantly with memory retention, either with or without correcting for initial memory performance or sleepiness (all Ps > $0.11)$.

In summary, our two main findings are: (1) spindle density is considerably higher during deep sleep than during light sleep, and (2) sleep spindle density during SWS is strongly and robustly associated with over-nap memory retention, while not at all during light sleep.

With regard to the first finding, we demonstrate a $>60 \%$ increase in spindle density during deep sleep relative to light sleep. At the same time, more fundamental spindle characteristics, such as duration and amplitude, did not change with sleep depth. Hence, elementary spindle physiology appears unaffected by sleep state. Higher spindle density during SWS is at odds with traditional literature that suggests spindles take place predominantly during light sleep. This discrepancy may be resolved by taking into account that these older findings rely on visual inspection for spindle detection. Automated approaches, like the one used here, are able to identify spindles occurring during highamplitude slow waves when spindles are not readily discernible by the human eye.

Our observed spindle densities are roughly similar to values obtained with other detection algorithms in both EEG (Gais et al. 2002; Mölle et al. 2011) and intracortical (Peter-Derex et al. 2012) studies, suggesting that our finding is not an artifact stemming from the spindle algorithm used. Nonetheless, the higher spindle density for SWS that we report is at variance with two other studies that differentiate between light and deep sleep spindle density and do not find such differences (Gais et al. 2002; Mölle et al. 2011). Various factors may contribute to these incon- sistencies, such as the much shorter sleep duration in our study (nap vs. full night of sleep), our subjects' partially sleepdeprived state, or circadian influences, which are known to strongly affect $\sigma$ power across the sleep-wake cycle (Dijk et al. 1997). At present, we cannot distinguish among these possibilities.

Our second main finding consists of a strong and specific correlation between declarative memory retention and spindle density in SWS. Such a correlation was to no extent apparent in stage 2 sleep. Direct comparison of deep and light sleep correlation coefficients also attested to a much stronger link between spindles and memory in deep sleep. Partial correlations revealed that this pattern of results was unrelated to sleepiness and initial memory performance.

In light of the previously observed coupling between slow oscillation phase and spindle occurrence (e.g., Mölle et al. 2009), as well as the demonstrated impact of slow oscillations on memory performance (e.g., Marshall et al. 2006), we believe our findings may reflect that sleep spindles are more effectively involved in memory consolidation when they occur in the presence of slow oscillations. In this scenario, while spindles take place during both light and deep sleep, their potentiating effects on neocortical synapses are largely restricted to the window provided by the slow oscillation up-state. Such limits on inducing plasticity could be related to cortical membrane potential levels, or neuromodulatory input to these areas that varies with slow oscillation phase (Eschenko et al. 2011).

Although direct support for a "window of potentiation" is lacking, findings of hippocampal ripple and cortical slow wave co-occurrence (Sirota et al. 2003) suggest neural replay may itself be preferably nested in spindles modulated by the slow oscillation, rather than in spindles occurring during light sleep. Other evidence indicates that spindles have a facilitating role in keeping a train of slow oscillations going (Mölle et al. 2011), thereby presumably promoting their own recurrence in the next slow oscillation cycle. This, in turn, would provide the opportunity for additional synaptic modification. Although we did not analyze slow oscillations or their grouping influence on spindles directly, we assume that this repeatedly demonstrated coupling phenomenon is present in our sample as well.

Of note, previous studies (e.g., Bódizs et al. 2005; Schabus et al. 2008) report a positive relation between spindles and general cognitive or learning abilities. In principle, this relation could underlie the reported association between spindle density and memory retention. Interpretation of our findings in these terms is highly unlikely, however, as the potential contribution from learning aptitude was effectively factored out by including initial memory performance as a covariate in the correlation analyses.

It should be mentioned that we do not claim that sleep spindles during light sleep are completely unrelated to memory processing, as various studies have demonstrated otherwise (e.g., Gais et al. 2002; Schabus et al. 2008; Tamaki et al. 2008). Rather, we provide specific evidence that declarative memory consolidation and rate of spindle occurrence during SWS, but not during light sleep, are coupled.

While spindles correlated strongly and robustly with memory retention, power measures, including power in the slow oscillation band and $\sigma$ range, did not. This suggests that, while both slow oscillations and sleep spindles likely play a role in memory 
consolidation, power measures may not always sufficiently reflect the functional relevance of these brain rhythms.

Sleep architecture parameters were not associated with memory retention either, which is in line with some studies (e.g., Clemens et al. 2006), but not others (e.g., Takashima et al. 2006). The absence of this link may be due to the limited sleep opportunity provided, or to the influence of the partial sleep deprivation on sleep architecture, both of which might have weakened or obscured a relation with memory retention. These explanations echo the point made above for power: Under the assumption that certain distinct electrophysiological events (i.e., sleep spindles) are functionally involved in systems memory consolidation, focusing on general sleep measures will dilute any effect present.

Finally, the correlational nature of our findings implies that no firm conclusions can be drawn regarding causality. Still, the strong coupling between spindles and memory retention we describe persisted when correcting for alternative explanatory factors. Combined with an increasing literature on the connection between spindles, slow oscillations, and memory, we are inclined to interpret our findings as indicating that SWS-related spindles play a central role in memory consolidation.

In conclusion, contrary to common notion, we find higher spindle density during deep than during light sleep. Most importantly, we provide novel evidence that sleep spindle prevalence during SWS, but not during light sleep, is related to offline consolidation of declarative memory. This could reflect that spindles are more effective at potentiating cortical networks when in the presence of attendant slow oscillations, than in their absence.

\section{Acknowledgments}

This work was funded by NWO grant 452-08-013.

\section{References}

Bódizs R, Kis T, Lázár AS, Havrán L, Rigó P, Clemens Z, Halász P. 2005. Prediction of general mental ability based on neural oscillation measures of sleep. J Sleep Res 14: 285-292.

Bonjean M, Baker T, Lemieux M, Timofeev I, Sejnowski T, Bazhenov M. 2011. Corticothalamic feedback controls sleep spindle duration in vivo. J Neurosci 31: 9124-9134.

Born J, Rasch B, Gais S. 2006. Sleep to remember. Neuroscientist 12: $410-424$.

Buzsáki G. 1996. The hippocampo-neocortical dialogue. Cereb Cortex 6: $81-92$.

Clemens Z, Fabó D, Halász P. 2005. Overnight verbal memory retention correlates with the number of sleep spindles. Neuroscience 132: $529-535$.

Clemens Z, Fabó D, Halász P. 2006. Twenty-four hours retention of visuospatial memory correlates with the number of parietal sleep spindles. Neurosci Lett 403: 52-56.

Clemens Z, Mölle M, Eröss L, Barsi P, Halász P, Born J. 2007. Temporal coupling of parahippocampal ripples, sleep spindles and slow oscillations in humans. Brain 130: $2868-2878$.

Clemens Z, Mölle M, Eröss L, Jakus R, Rásonyi G, Halász P, Born J. 2011. Fine-tuned coupling between human parahippocampal ripples and sleep spindles. Eur J Neurosci 33: 511-520.

Dijk DJ, Shanahan TL, Duffy JF, Ronda JM, Czeisler CA. 1997. Variation of electroencephalographic activity during non-rapid eye movement and rapid eye movement sleep with phase of circadian melatonin rhythm in humans. J Physiol 505: 851-858.

Eschenko O, Magri C, Panzeri S, Sara SJ. 2011. Noradrenergic neurons of the locus coeruleus are phase locked to cortical up-down states during sleep. Cereb Cortex 22: 426-435.

Ferrarelli F, Huber R, Peterson MJ, Massimini M, Murphy M, Riedner BA, Watson A, Bria P, Tononi G. 2007. Reduced sleep spindle activity in schizophrenia patients. Am J Psychiatry 164: 483-492.

Gais S, Mölle M, Helms K, Born J. 2002. Learning-dependent increases in sleep spindle density. J Neurosci 22: 6830-6834.

Marshall L, Helgadóttir H, Mölle M, Born J. 2006. Boosting slow oscillations during sleep potentiates memory. Nature 444: 610-613.

Marshall L, Kirov R, Brade J, Mölle M, Born J. 2011. Transcranial electrical currents to probe EEG brain rhythms and memory consolidation during sleep in humans. PLOS One 6: e16905. doi: 10.1371/journal. pone.0016905.

Meng X, Rosenthal R, Rubin DB. 1992. Comparing correlated correlation coefficients. Psychol Bull 111: 172-175.

Mölle M, Marshall L, Gais S, Born J. 2002. Grouping of spindle activity during slow oscillations in human non-rapid eye movement sleep. J Neurosci 22: 10941-10947.

Mölle M, Eschenko O, Gais S, Sara SJ, Born J. 2009. The influence of learning on sleep slow oscillations and associated spindles and ripples in humans and rats. Eur J Neurosci 29: 1071-1081.

Mölle M, Bergmann TO, Marshall L, Born J. 2011. Fast and slow spindles during the sleep slow oscillation: disparate coalescence and engagement in memory processing. Sleep 34: 1411-1421.

O’Neill J, Pleydell-Bouverie B, Dupret D, Csicsvari J. 2010. Play it again: reactivation of waking experience and memory. Trends Neurosci 33: 220-229.

Peter-Derex L, Comte JC, Mauguière F, Salin PA. 2012. Density and frequency caudo-rostral gradients of sleep spindles recorded in the human cortex. Sleep 35: 69-79.

Rechtschaffen A, Kales A. 1968. A manual of standardized terminology, techniques and scoring system for sleep stages of human subjects. Neurological Information Network, Bethesda, MD.

Rosanova M, Ulrich D. 2005. Pattern-specific associative long-term potentiation induced by a sleep spindle-related spike train. J Neurosci 25: $9398-9405$.

Saletin JM, Goldstein AN, Walker MP. 2011. The role of sleep in directed forgetting and remembering of human memories. Cereb Cortex 21: 2534-2541.

Schabus M, Hoedlmoser K, Pecherstorfer T, Anderer P, Gruber G, Parapatics S, Sauter C, Kloesch G, Klimesch W, Saletu B, et al. 2008. Interindividual sleep spindle differences and their relation to learning-related enhancements. Brain Res 1191: 127-135.

Schmidt C, Peigneux P, Muto V, Schenkel M, Knoblauch V, Münch M, de Quervain DJ, Wirz-Justice A, Cajochen C. 2006. Encoding difficulty promotes postlearning changes in sleep spindle activity during napping. J Neurosci 26: 8976-8982.

Sirota A, Csicsvari J, Buhl D, Buzsáki G. 2003. Communication between neocortex and hippocampus during sleep in rodents. Proc Natl Acad Sci 100: $2065-2069$.

Steriade M. 2006. Grouping of brain rhythms in corticothalamic systems. Neuroscience 137: 1087-1106.

Takashima A, Petersson KM, Rutters F, Tendolkar I, Jensen O, Zwarts MJ, McNaughton BL, Fernández G. 2006. Declarative memory consolidation in humans: a prospective functional magnetic resonance imaging study. Proc Natl Acad Sci 103: 756-761.

Tamaki M, Matsuoka T, Nittono H, Hori T. 2008. Fast sleep spindle (13$15 \mathrm{~Hz}$ ) activity correlates with sleep-dependent improvement in visuomotor performance. Sleep 31: 204-211.

Van der Helm E, Gujar N, Nishida M, Walker MP. 2011. Sleep-dependent facilitation of episodic memory details. PLOS One 6: e27421. doi: 10.1371 /journal.pone.0027421.

Received March 9, 2012; accepted in revised form April 27, 2012. 


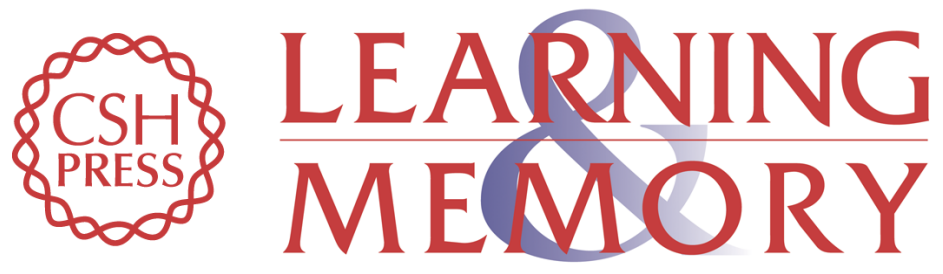

\section{Involvement of spindles in memory consolidation is slow wave sleep-specific}

Roy Cox, Winni F. Hofman and Lucia M. Talamini

Learn. Mem. 2012, 19:

Access the most recent version at doi:10.1101//m.026252.112

References This article cites 29 articles, 7 of which can be accessed free at:

http://learnmem.cshlp.org/content/19/7/264.full.html\#ref-list-1

License

Email Alerting Receive free email alerts when new articles cite this article - sign up in the box at the Service top right corner of the article or click here. 